\title{
Modified Exemplar based Image Inpainting Algorithm
}

\author{
Vijayalakshmi A. \\ Dept. of Computer Science \\ Bangalore, India
}

\author{
Pethuru Raj, PhD \\ Cloud Architect \\ IBM India Bangalore
}

\begin{abstract}
Recovering lost part of an image plays a great role in image processing. Inpainting is a technique that helps in recovering lost pixels from an image. From the existing techniques of Inpainting, Exemplar Inpainting is one of the fast and better techniques that help in restoring the lost part of an image. Exemplar based method chooses a patch similar to the lost patch from the known area to fill in the occluded surface. This paper proposes a modified exemplar based Inpainting algorithm for restoring the unknown pixels in a lost region in an image. In the proposed work, the pixel at the contour of the missing region is prioritized by taking into consideration the most known pixels around. In order to find the strength of a pixel in this proposed approach, a Sobel detector is used and the magnitude of the pixel is found. Further the most similar patch to the unknown region is searched in the known area using structural similarity index. The experimental result shows that this method shows an improvement in filling the lost region by finding the exact match from the known area of the given image.
\end{abstract}

\section{Keywords}

Inpainting, Exemplar Inpainting, Image inpainting, Patch based.

\section{INTRODUCTION}

Active research in face recognition is increasing these days because of the major fact that face recognition requires less intervention from the user. Face recognition based on video is a great advantage to the world in terms of security. The major factors that act as a drawback to the face recognition are illumination, pose variation and occlusion under uncontrolled scenarios. These factors decrease the recognition rate in identifying faces. Among these factors partial occlusion is a major component that decreases the recognition rate of faces in uncontrolled situations. This leads to the fact that when a face recognition system is constructed, the system should be robust to partial occlusion.

Recent studies shows that to recover these occluded region, Inpainting technique can be used. Image inpainting is a technique used in old ages to restore the lost part of an image. This technique helps in restoring the lost region of an image from information obtained from surrounding regions [1]. To restore the part of the image that is occluded, information can be taken from region outside the part that is occluded. There are various applications of image inpainting. Removal of scratches from old photos, recovering lost region of an image is to name a few.

Research in this area of image inpainting, classifies the inpainting techniques into

1. Texture synthesis based inpainting

2. Partial differential based inpainting

3. Exemplar and search based inpainting

\subsection{Texture Synthesis}

Texture synthesis is a method used to fill in small regions with pattern similar to the given sample. In the case of texture synthesis, for a missing region a texture is been synthesized by each pixel by pixel. This particular technique can address a small portion of inpainting technique alone as they are not suitable for synthesizing missing regions of a curved image [2]. This method of texture synthesis assumes large sample from which texture can be drawn. In this method the block inclusive of the missing region is replaced with the best matched block from the neighborhood. In the work carried out in [3] proposes an algorithm that is designed which synthesizes a texture from an existing large image based on blocks using non parametric approach. The nearest matching block is selected using conditional probability distribution and sampled from this. This algorithm produces good result for a varied range of textures.

\subsection{Partial Differential Based Inpainting}

Partial Differential based inpainting is a geometry oriented method. In this approach, images are modeled as function with degrees of smoothness. Interpolation is performed using partial differential equation (PDE) [4]. The methods that use geometry oriented approaches works well with only local data/ neighboring pixels around the occluded region. This method helps in spreading the contour information along isophote direction with the technique of diffusion.

\subsection{Exemplar and Search based Inpainting}

In contrast to geometry oriented inpainting technique, exemplar inpainting is texture oriented. This method was developed as an application to texture synthesis. A work carried out in [3] authors proposed a probabilistic approach of synthesizing data. There are many exemplar algorithms that are designed where the visible part of the image is used as a source to fill the occluded region. In this method compared to texture synthesis, the missing region is replicated rather than synthesizing data from the information available. The major drift in this approach was the work proposed by Criminisi [5], which combined the PDE and texture synthesis method to fill the occluded region choosing a similar patch from the known region.

In general, exemplar based inpainting algorithm is composed of following steps [6].

1. Initial occluded region is detected and are represented with the data structures that are appropriate to the region.

2. A predefined function is used to compute the filling order for all the missing pixels as the first step in each of the iterations.

3. From the source region, the most matching block is searched to fix the given occluded regions block with a pixel cantered at $\mathrm{p}$.

4. Boundary of the target region and the information for computing filling priorities are updated. 


\section{RELATED WORK}

Exemplar based image inpainting works better when compared to PDE and texture synthesis methods. Criminisi [5] combined PDE and texture synthesis to provide a better inpainting technique to fill in the hole created in an image. There has been research going on improving this approach that helps in more accurate fill of the occluded region with the patch obtained from the available region of an image.

One of the drawbacks of Criminisi's approach is that of high time cost. This is reduced using a search strategy in [6]. In this work the authors claim that most of the similar patch that matches with the target patches lies around the target patch and they propose a concept of window. A window length is denoted manually for each window. Window length and actual window size is calculated as

Window length $=$ steplength $*$ patchsize

$(2 *$ windowlength +1$) *(2 *$ windowlength +1$)$ respectively. This change in defining the window size forces the search for the similar patch in the local area and reduces the cost of computation. Along with this instead of using SSD (Sum of Square Distance), L1Euclidean Distance Norm is used to compute the similarity of the patches. Once the most similar patch is chosen, the pixels are filled in the target region using color transfer method instead of direct fill as in the case with Criminisi's approach. Mean and standard deviation for both the target patch $\Psi_{\mathrm{p}}$ and the most matched patch from the source region, $\Psi_{\mathrm{q}}$ is computed. The mean is subtracted from the pixels selected and these are scaled by respective standard deviation. To get the transferred image patch, the average computed for the target images is added. The authors claim the effectiveness of the proposed method through experiments.

Wen-Huang Cheng et. al in their work of proposing a robust algorithm for exemplar image inpainting, [7] which identifies a dropping effect of the confidence value to zero with respect to Criminisi's algorithm. The authors modified the priority function from a multiplicative form to additive form as

$\mathrm{P}(\mathrm{p})=\mathrm{C}(\mathrm{p})+\mathrm{D}(\mathrm{p})$ where $\mathrm{C}(\mathrm{p})$ is the confidence term and $\mathrm{D}(\mathrm{p})$ is the data term. Criminisi algorithm work good for linear structures. In order accept it for the curved structures, the authors propose a generalized function $\mathrm{RC}(\mathrm{p})$ to smoothen the curve of the confidence term $\mathrm{C}(\mathrm{p})$ to match with the data term with $\omega$ as the regularizing factor for the case with curves is given as,

$\mathrm{RC}(\mathrm{p})=(1-\omega) * \mathrm{C}(\mathrm{p})+\omega$ where $0<=\omega<=1$

Authors claim that the above priority function avoids noises and is robust to the phenomena of filling the missing region in an image.

An attempt is made to improve the maintenance of linear edges during the filling of the pixels in the target area in [8]. In Criminisi algorithm, the confidence term and the data term are used in identifying the patch to be filled, where as in the proposed work authors modified the priority function as,

$P(p)=C(p) \exp \left(\frac{D(p)}{2 \sigma^{2}}\right)$

In addition to this, to select similar patch from the contour region and the known area, a new metric is chosen in this method as a combination of SSD (Sum of Square Distance) and Hellinger distance. This metric proposed by the authors in this work, carries the whole information from the candidate patches. Authors claim that this metric helps in selecting more visually pleasant patches for filling in the missing region.
In [9] a modified exemplar image inpainting algorithm is proposed. In this method, authors feel that measuring similarity of two patches based only on color is not enough to diffuse the structure into the target region that is to filled. Hence a new function, image gradient is added to this function to get the value from

$\mathrm{G}=\mathrm{G}\left(\Psi_{\mathrm{p}}-\Psi_{\mathrm{q}}\right)$

Where, G defines each pixel's gradient value in both the patches. Including the gradient value into the function, the similarity function is based on the difference in color and the gradient value of the pixels. Magnitude and direction of the gradient provides information like how quickly the color changes and in which direction it is changing. Experimental result with this modification in the exemplar based image inpainting algorithm shows enhance in the performance.

Drori et al [9] in their work, missing image is synthesized. In this work, the occluded region is iteratively by image fragments using the principle of self-similarity. The fragment of image is selected from most similar set of fragments. The results show a good performance in filling but the computational time is long compared to existing methods.

\section{EXEMPLAR BASED INPAINTING}

In this section we provide a detailed explanation on Criminisi approach of image inpainting. One of the important works in Exemplar based image inpainting is carried out by Criminisi [5]. This algorithm combines the advantage of texture synthesis method and PDE-based inpainting method. The method proposed in this work consists of the following major steps for filling the missing region.

1. Counts the known pixels that belongs to the edge and perform edge detection.

2. As a second step, the distance is computed between the patch of missing region and the entire known region is calculated.

Figure 1 gives an introduction to the terms used in the algorithm.

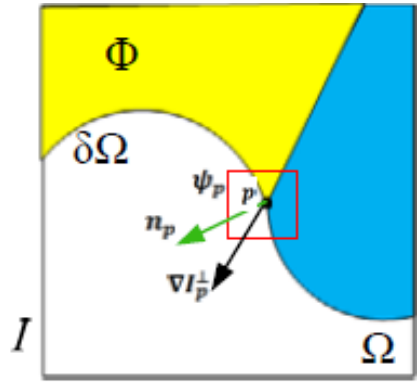

Fig 1: Demonstration of the terms used to represent elements of occluded region and known region.

Consider I as the whole image that includes both the known region and the occluded region that is to be filled. Let $\Omega$ represent the target region where the pixels are missing and need to be filled. $\Phi$ represents the source region from which a most similar patch is selected to fill the target region. $\delta \Omega$ represent the contour of the region to be filled. This can also be known as fill front. The pixels are selected from the fill front and are diffused inwards.

Consider the following figure, Figure 2 that clearly explains the procedure followed in filling pixels using the above method. 

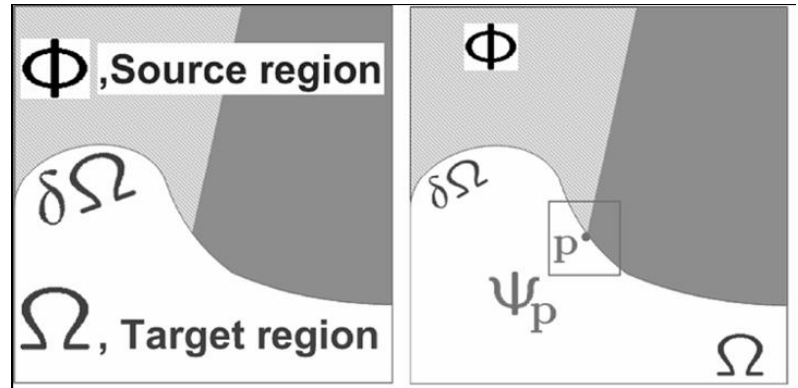

a

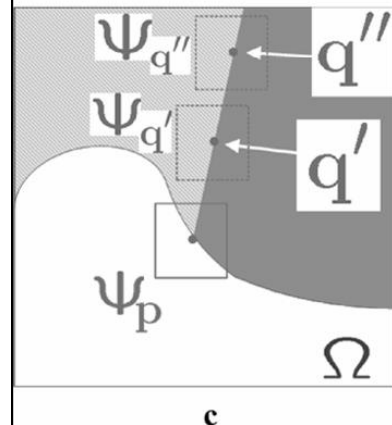

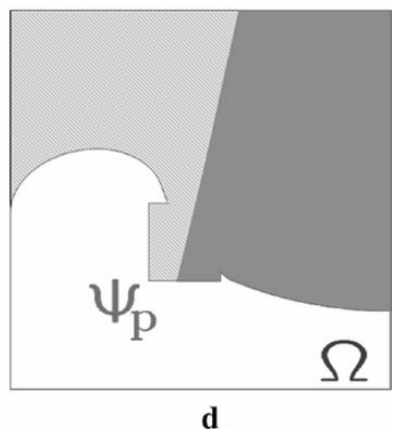

Fig 2: Basic steps to show the process of filling the occluded region using Criminisi's algorithm.

The square template $\Psi_{\mathrm{p}}$ is the patch centered at $\mathrm{p}$, belongs to $\Omega$ whose center is at $P$ that is to be filled. If $\Psi_{p}$ lies in the continuation of an edge, the most similar match $\Psi_{\mathrm{q}} \in \Phi$ also lies in the same edge.

\subsection{Procedure of Inpainting}

From the given input image in Figure 2 (a) the target region $\Omega$ is selected. The source region $\Phi$ is the whole image that is I minus the target region, $\Omega$.

$\Phi=\mathrm{I}-\Omega$

The patch $\Psi$ is to be specified with a size. In this method the size is considered as the default size $9 \mathrm{x} 9$ pixels. According to the algorithm each pixel maintains a colour value and a confidence value. The colour value will be empty if the pixel is not filled. The confidence value is fixed once the pixel is filled. The pixels along the fill front (contour) are given temporary priority value to determine the order of filling. The algorithm consists of three basic steps on which iteration follows.

\section{Computation of patch priority}

The priority is given to each pixel on the fill front based on if the pixel is a continuation of the strong edge and if they are surrounded by high confidence pixels.

For a patch $\Psi_{\mathrm{p}}$ with center at $\mathrm{p}$ for any $\mathrm{p} \in \delta \Omega$, the priority $\mathrm{P}(\mathrm{p})$ of that pixel is set with the equation,

$\mathrm{P}(\mathrm{p})=\mathrm{C}(\mathrm{p}) \mathrm{D}(\mathrm{p})$

Where $C(p)$ is the confidence term and $D(p)$ is defined as the data term and given as,

$$
\begin{gathered}
\mathrm{C}(\mathrm{p})=\sum \frac{q \in \varphi_{p} \cap \emptyset C(q)}{\left|\varphi_{p}\right|}, 0 \leq C \leq 1 \\
D(p)=\frac{\nabla I_{p}{ }^{\prime} \cdot n_{p}}{\alpha}
\end{gathered}
$$

In the above equation, $\left|\Psi_{\mathrm{p}}\right|$ designates the area of the patch $\Psi_{\mathrm{p}}$ and $\alpha$ is the normalization factor. $n_{p}$ denotes a unit vector orthogonal to the front $\delta \Omega$ in the point at pixel p. Ip' signifies the orthogonal operator. With the above equation the priority value $\mathrm{P}(\mathrm{p})$ for each pixel on the contour is calculated.

The value of

$\mathrm{C}(\mathrm{p})=0$ for all $\mathrm{p} \in \Omega$

$\mathrm{C}(\mathrm{p})=1$ for all $\mathrm{p} \in \mathrm{I}-\Omega$

$\mathrm{C}(\mathrm{p})$ is a confidence term that gives information of the surrounding pixels around $\mathrm{P}$. Those patches that have more of the pixels around it available are the patches that are prioritized for filling. The data term D (p) defines the strength of the isophote that are selected at $\delta \Omega$ at each of the iteration in the filling process. Hence this will enhance the priority of a patch. This will help the linear structure to be synthesized first in the filling process.

2. Structure Information

Once the priorities of the pixels in the fill front are computed, the patch with highest priority is found as $\Psi_{\mathrm{p}}$, This patch is filled with the patch that is matched from the source region $\Phi$. The most similar patch is searched in the source as

$$
\varphi_{q^{\prime}}=\arg \min _{\varphi_{q} \in \varnothing} d\left(\varphi_{p^{\prime}}, \varphi_{q}\right)
$$

Here the distance between $\Psi_{\mathrm{p}}$ and $\Psi_{\mathrm{p}}$, are calculated using sum of square differences(SSD).

Once the source patch is found, the value of the pixel that is to be filled in the target region say p' where

$P^{\prime} \in \varphi_{p \cap \Omega}$

is taken from its corresponding position of the source patch $\Psi_{\mathrm{p}}$,

$$
C(p)=C\left(p^{\prime}\right) \forall p \in \Psi_{p^{\prime}} \cap \Omega
$$

\subsection{Comparative analysis of exemplar based Inpainting algorithm}

Exemplar based image inpainting is considered to be one of the best inpainting technique for filling in the occlusion in an image. Criminisi proposed a combination of PDE and texture synthesis to fill in the missing region of an image. With Criminisi as the base algorithm there are modification done to improve performance with respect to the above algorithm. Table 1 provides a comparative study on the various methods proposed in exemplar based image inpainting algorithm.

Table 1. Comparative study on the limitations of exemplar based image inpainting algorithms.

\begin{tabular}{|c|c|}
\hline Algorithm & Limitations \\
\hline Criminisi & $\begin{array}{c}\text { This method is not appropriate for } \\
\text { handling curved structure of an image. }\end{array}$ \\
\hline W.Cheng & $\begin{array}{c}\text { This method cannot handle complex } \\
\text { geometrical structure }\end{array}$ \\
\hline
\end{tabular}




\begin{tabular}{|c|c|}
\hline Jason & $\begin{array}{c}\text { Efficiency depends upon the Bezier } \\
\text { curve, this method cannot handle } \\
\text { geometrical structure }\end{array}$ \\
\hline Anupam & $\begin{array}{c}\text { Does not work with videos. } \\
\text { Computational complexity has to be } \\
\text { improved. }\end{array}$ \\
\hline Manali Desai & Edge continuity is missing \\
\hline $\begin{array}{c}\text { R. Mart'inez- } \\
\text { Noriega, A. } \\
\text { Roumy }\end{array}$ & $\begin{array}{c}\text { This method does not provide good } \\
\text { result on comparison with state of art } \\
\text { methods on curved surfaces. }\end{array}$ \\
\hline
\end{tabular}

\section{MODIFIED APPROACH}

In the proposed work, the criminisi algorithm is modified in finding the priority of the best pixel on the contour and further finding the proper match among the known pixels.

\section{Proposed algorithm}

Step 1: The target region initialization. In this step, the missing areas in the image are identified.

Step 2: Computation of patch priority

The priority is given to each pixel on the fill front based on if the pixel is a continuation of the strong edge and if they are surrounded by high confidence pixels.

For a patch $\Psi p$ with center at $p$ for any $p \in \delta \Omega$, the priority $\mathrm{P}(\mathrm{p})$ of that pixel is set with the equation,

$\mathrm{P}(\mathrm{p})=\mathrm{C}(\mathrm{p}) \mathrm{D}(\mathrm{p})$

Where $C(p)$ is the confidence term and $D(p)$ is defined as the data term. The value of $\mathrm{C}(\mathrm{P})$ is initialized at the beginning of the algorithm as:

$\mathrm{C}(\mathrm{p})=0$ for all $\mathrm{p} \in \Omega$

$\mathrm{C}(\mathrm{p})=1$ for all $\mathrm{p} \in \mathrm{I}-\Omega$

$\mathrm{C}(\mathrm{p})$ is a confidence term that gives information of the surrounding pixels around $\mathrm{P}$. Those patches that have more of the pixels around it available are the patches that are prioritized for filling.

$\mathrm{C}(\mathrm{p})$ is calculated as

$C(p)=\frac{\sum q \in \varphi_{p} \cap \varnothing C(q)}{\left|\varphi_{p}\right|}, 0 \leq C \leq 1$

In the above equation, $|\Psi \mathrm{p}|$ designates the area of the patch. In this case a $3 \times 3$ matrix is considered for the patch. To calculate the data term, Sobel edge detector is used to find the strength of the pixel on the edge. As larger mask reduce the error rate, in this case a mask of 2 is used with $c=2$ [12].This leads to a Sobel operator,

$$
\mathrm{M}_{\mathrm{x}}=\begin{array}{rrr}
-1 & 0 & 1 \\
-2 & 0 & 2 \\
-1 & 0 & 1
\end{array} \text { and } \mathrm{M}_{\mathrm{y}}=\begin{array}{rcc}
-1 & -2 & -1 \\
0 & 0 & 0 \\
1 & 2 & 1
\end{array}
$$

The major steps in finding the magnitude of the gradient are as given in Table 2.
Table 2.Detecting edges using masks.

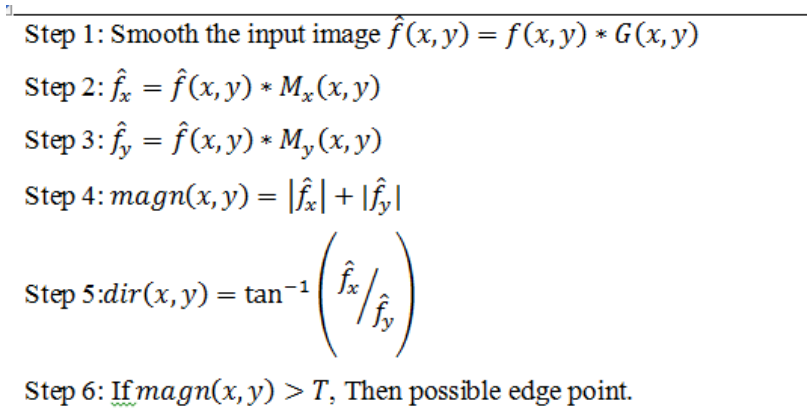

Once the strength of the pixel is found, it signifies the most prioritized patch on the edge.

Step 3: For every patch, the most similar patch from the source region is selected. This is done on the basis of structural similarity measure (SSIM).

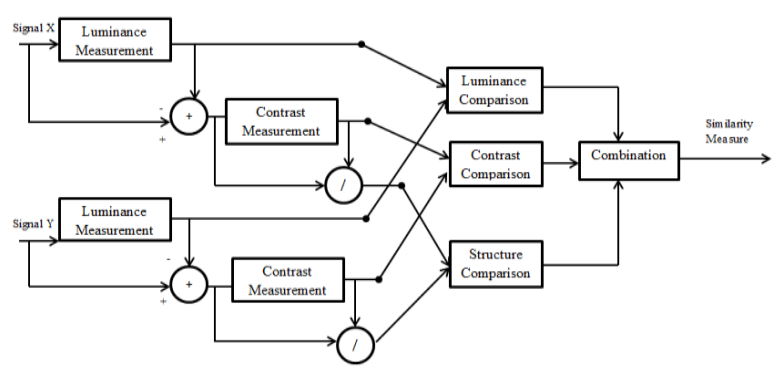

Fig 3: Diagrammatical representation of the working of structural similarity measure.

Figure 3 [10] explains the system diagram of similarity measurement. Considering $\mathrm{x}$ and $\mathrm{y}$ to be the image signals, from the target patch and the patch considered for match. The similarity index can serve as the quantitative measure for the quality of the second signal. The task of similarity index is divided into three different comparisons: luminance, contrast and structure. As a first step, luminance of each of the signal is compared followed by removing the intensity from the signal and further signal is normalized by its own standard deviation. Structure comparison is been calculated on the normalized signals. Overall similarity measure is calculated combining the above three components.

Step 4: Searching for the best patch starts from the patch of pixels around the target patch saving the computational time for patch selection.

Step 5: Replace the target patch with the most similar patch identified.

\section{EXPERIMENTAL RESULT}

Inorder to check the efficiency of the proposed method, the algorithm is compared with criminisi algorithm that uses mean square for selection of most similar patch. 

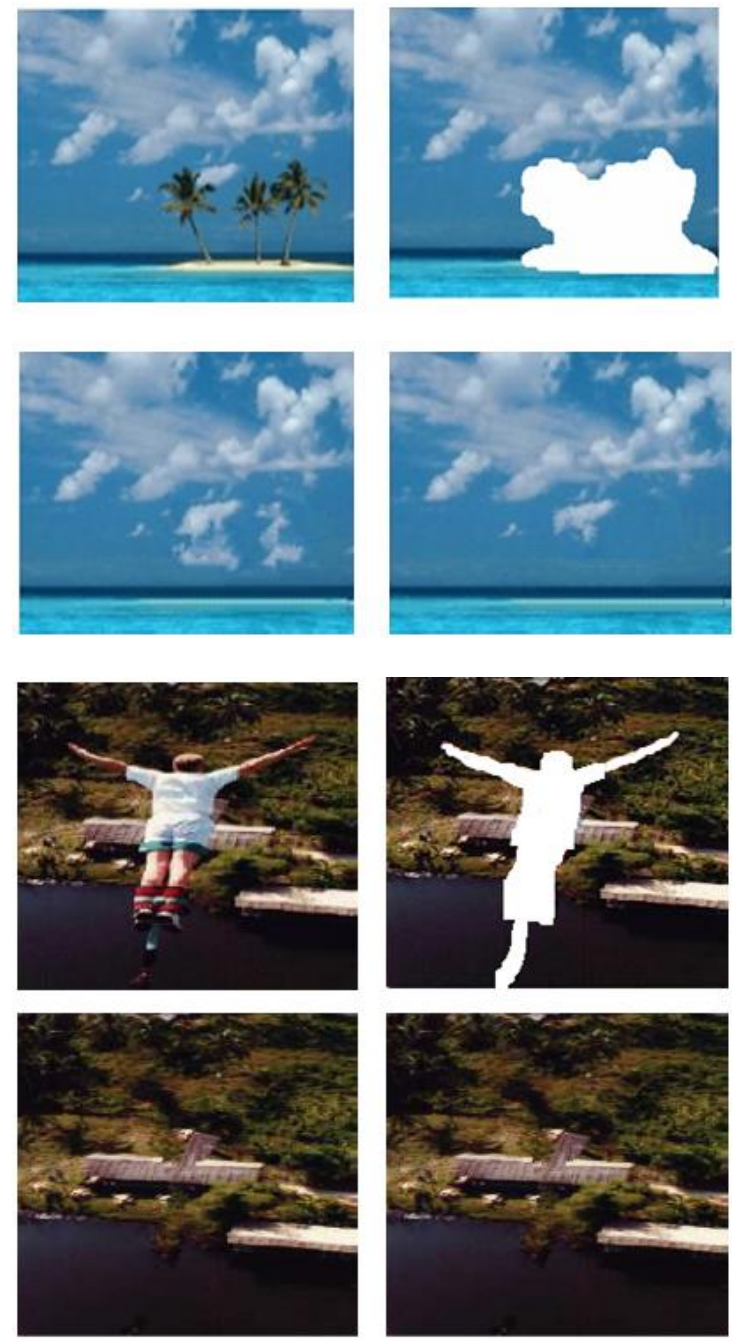

Fig 4: Original image, Image for filling the missing region, Criminisi Algorithm, Proposed method (clockwise).

\section{CONCLUSION}

In this work, we presented a modified method for exemplar based image inpainting and this modification lead to a better improvement in producing better result. This method uses structural similarity measure for matching the best patches and patches are searched for the best match from the center pixel of the target pixel. This reduces the computational time in searching for the best match. The algorithm is applied on images and compared the result with respect to Criminisi method.

\section{REFERENCES}

[1] Ravi, S., Pasupathi, P., Muthukumar, S. and Krishnan, N., 2013, March. Image in-painting techniques-A survey and analysis. In Innovations in Information Technology (IIT), 2013 9th International Conference on (pp. 36-41). IEEE.

[2] Chavda, P. and Gagnani, L., 2015. Survey on Image Inpainting Techniques: Texture Synthesis, Convolution and Exemplar Based Algorithms. International Journal for Innovative Research in Science and Technology, 1(7), pp.100-106.

[3] Efros, A. and Leung, T.K., 1999. Texture synthesis by non-parametric sampling. In Computer Vision, 1999. The Proceedings of the Seventh IEEE International Conference on (Vol. 2, pp. 1033-1038). IEEE.

[4] Arias, P., Facciolo, G., Caselles, V. and Sapiro, G., 2011. A variational framework for exemplar-based image inpainting. International journal of computer vision, 93(3), pp.319-347.

[5] Criminisi, A., Pérez, P. and Toyama, K., 2004. Region filling and object removal by exemplar-based image inpainting. Image Processing, IEEE Transactions on, 13(9), pp.1200-1212.

[6] Chen, Q., Zhang, Y. and Liu, Y., 2007. Image inpainting with improved exemplar-based approach. In Multimedia Content Analysis and Mining (pp. 242-251). Springer Berlin Heidelberg.

[7] Cheng, W.H., Hsieh, C.W., Lin, S.K., Wang, C.W. and Wu, J.L., 2005, July. Robust algorithm for exemplarbased image inpainting. In The International Conference on Computer Graphics, Imaging and Vision (CGIV 2005) (pp. 64-69).

[8] Martínez-Noriega, R., Roumy, A. and Blanchard, G., 2012, September. Exemplar-based image inpainting: Fast priority and coherent nearest neighbor search. In Machine Learning for Signal Processing (MLSP), 2012 IEEE International Workshop on (pp. 1-6). IEEE.

[9] Patel, A.G., Kumar, S. and Prajapati, A.D., 2014. Improved Exemplar based Image Inpainting using Structure Tensor. International Journal of Computer Applications, 96(15).

[10] Drori, I., Cohen-Or, D. and Yeshurun, H., 2003, July. Fragment-based image completion. In ACM Transactions on Graphics (TOG) (Vol. 22, No. 3, pp. 303-312). ACM.

[11] Wang, Z., Bovik, A.C., Sheikh, H.R. and Simoncelli, E.P., 2004. Image quality assessment: from error visibility to structural similarity. Image Processing, IEEE Transactions on, 13(4), pp.600-612.

[12] Vincent, O. R., and Olusegun Folorunso. "A descriptive algorithm for sobel image edge detection." In Proceedings of Informing Science \& IT Education Conference (InSITE), vol. 40, pp. 97-107. 2009. 